\title{
BMJ Open Renal hypothermia during partial nephrectomy for patients with renal tumours: a randomised controlled clinical trial protocol
}

Rodney H Breau, ${ }^{1,2}$ Ilias Cagiannos, ${ }^{1}$ Greg Knoll, ${ }^{2}$ Christopher Morash, ${ }^{1}$ Sonya Cnossen, ${ }^{2}$ Luke T Lavallée, ${ }^{1,2}$ Ranjeeta Mallick, ${ }^{2}$ Antonio Finelli, ${ }^{3}$ Michael Jewett, ${ }^{3}$ Bradley C Leibovich, ${ }^{4}$ Jonathan Cook, ${ }^{5}$ Louise LeBel, ${ }^{2}$ Anil Kapoor, ${ }^{6}$ Frederic Pouliot, ${ }^{7}$ Jonathan Izawa, ${ }^{8}$ Ricardo Rendon, ${ }^{9}$ Dean A Fergusson ${ }^{2}$

To cite: Breau RH,

Cagiannos I, Knoll G, et al. Renal hypothermia during partial nephrectomy for patients with renal tumours: a randomised controlled clinical trial protocol. BMJ Open 2019;9:e025662. doi:10.1136/ bmjopen-2018-025662

- Prepublication history for this paper is available online. To view these files, please visit the journal online (http://dx.doi. org/10.1136/bmjopen-2018025662).

Received 25 July 2018 Revised 10 October 2018 Accepted 12 0ctober 2018
Check for updates

(C) Author(s) (or their employer(s)) 2019. Re-use permitted under CC BY-NC. No commercial re-use. See rights and permissions. Published by BMJ.

For numbered affiliations see end of article.

Correspondence to Dr Rodney H Breau; rbreau@toh.on.ca

\section{ABSTRACT}

Introduction Partial nephrectomy is a standard of care for non-metastatic renal tumours when technically feasible. Despite the increased use of partial nephrectomy, intraoperative techniques that lead to optimal renal function after surgery have not been rigorously studied. Clamping of the renal hilum to prevent bleeding during resection causes temporary renal ischaemia. The internal temperature of the kidney may be lowered after the renal hilum is clamped (renal hypothermia) in an attempt to mitigate the effects of ischaemia. Our objective is to determine if renal hypothermia during open partial nephrectomy results in improved postoperative renal function at 12 months following surgery as compared with warm ischaemia (no renal hypothermia).

Methods and analyses This is a multicentre, randomised, single-blinded controlled trial comparing renal hypothermia versus no hypothermia during open partial nephrectomy. Due to the nature of the intervention, complete blinding of the surgical team is not possible; however, surgeons will be blinded until the time of hilar clamping. Glomerular filtration will be based on plasma clearance of a radionucleotide, and differential renal function will be based on renal scintigraphy. The primary outcome is overall renal function at 12 months measured by the glomerular filtration rate (GFR). Secondary outcomes include change in GFR, GFR of the affected kidney, change in GFR of the affected kidney, serum creatinine, haemoglobin, spot urine albumin to creatinine ratio, quality of life and postoperative complications. Data will be collected at baseline, immediately postoperatively and at 3, 6, 9 and 12 months postoperatively.

Ethics and dissemination Ethics approval was obtained for all participating study sites. Results of the trial will be submitted for publication in a peer-reviewed journal. Trial registration number NCT01529658; Pre-results.

\section{INTRODUCTION}

The diagnosis of renal tumours has increased by approximately $2 \%$ per year for over 30 years. ${ }^{1}$ Many new diagnoses are incidental
Strengths and limitations of this study

- Broad eligibility criteria of patients undergoing open partial nephrectomy.

- Detailed preoperative and postoperative renal function (glomerular filtration rate) assessment using plasma clearance and renal scintigraphy.

- Surgeons blinded to treatment allocation until just prior to renal artery clamping.

- The exclusion of patients treated with laparoscopic partial nephrectomy may limit generalisability.

due to more frequent use of abdominal imaging, ${ }^{2}$ and in 2017, it is estimated that over 63000 new renal cancers will be diagnosed in the USA. ${ }^{3}$ The increased detection of benign and malignant renal masses has resulted in a dramatic increase in the number of renal surgeries performed per year. ${ }^{4}$

Several institutions have reviewed their surgical series and reported improved renal function preservation and similar cancer outcomes for patients who receive partial nephrectomy (removing the tumour from the affected kidney) compared with radical nephrectomy (removing the entire affected kidney). ${ }^{5-12}$ It has also been suggested that partial nephrectomy patients have improved overall survival compared with radical nephrectomy patients, ${ }^{13}$ presumably because of fewer adverse effects as a result of renal insufficiency. ${ }^{14}$ These insights have resulted in a dramatic shift in the treatment of small renal masses. In a recent review of Canadian tertiary care institutions, the vast majority of tumours less than $7 \mathrm{~cm}$ in diameter are treated with partial nephrectomy. ${ }^{15}$ The urological community now recognises the importance of renal function preservation, and for this 
reason, partial nephrectomy is the preferred treatment of renal neoplasms when technically possible. ${ }^{16-18}$

Significant blood flow to the kidney necessitates temporary occlusion (clamping) of the renal hilar blood vessels to allow for tumour resection and precise renal reconstruction. ${ }^{19}$ As a consequence, the entire kidney is exposed to a period of ischaemia during partial nephrectomy. Ischaemia causes renal parenchymal injury and longer ischaemia time is associated with more severe renal damage. ${ }^{2021}$ Cooling the kidney (renal hypothermia) may decrease the metabolic demand of nephrons, which in turn may decrease acute ischaemic injury and long-term renal function. ${ }^{22-24}$ Ischaemic tissue hypothermia has also been used to reduce brain damage during circulatory arrest or in the period after cerebral infarction. ${ }^{2526}$ To achieve renal cooling, the kidney requires approximately 10 min surrounded by ice slush prior to tumour resection. Therefore, hypothermia necessitates a longer period of ischaemia that may be deleterious to recovery of renal function. The beneficial effect of hypothermia may be negated by the longer duration of ischaemia required.

Our objective is to determine if renal hypothermia during partial nephrectomy results in improved postoperative renal function compared with warm ischaemia (no hypothermia). Our primary objective is to determine the effect of hypothermia compared with no hypothermia on preservation of overall renal function at 12 months, as measured by glomerular filtration rate (GFR). Our secondary objective is to determine the effect of hypothermia compared with no hypothermia on preservation of renal function of the affected kidney (kidney with the tumour), as measured by the product of GFR and ipsilateral renal function.

\section{METHODS AND ANALYSES}

\section{Study setting and design}

This study was initiated and coordinated at The Ottawa Hospital, a regional cancer referral centre in Ottawa, Canada. Six urological oncology centres in Canada are participating in the trial (The Ottawa Hospital, University Health Network (Toronto), St. Joseph's Hospital (Hamilton), Centre Hospitalier Universitaire de Québec (Quebec City), London Health Sciences Centre and Capital District Health Authority (Halifax)). All study sites are tertiary referral centres that routinely perform open partial nephrectomy. Patients were randomised to receive either renal hypothermia (intervention) or no renal hypothermia (control).

Evaluation and flow of patients prior to, during and following surgery is according to the usual standard of care. The study flow chart is presented in figure 1 , and timeline of study/outcome assessments is outlined in table 1 .

\section{Eligibility criteria}

Study population

Patients undergoing open partial nephrectomy with renal hilar clamping. In keeping with a pragmatic approach to this study, eligibility criteria are inclusive, allowing for wide generalisability of results.

\section{Inclusion criteria}

Consenting adult patients ( $\geq 18$ years) presenting with a renal mass deemed amenable to an open partial nephrectomy with renal hilar clamping regardless of stage, histology, presence of solitary kidney, multiple tumours or baseline renal function.

\section{Exclusion criteria}

Patients are excluded from enrolment if they (1) present with a renal mass deemed appropriate for open partial nephrectomy that does not require renal hilar clamping, (2) have bilateral renal tumours with planned surgery, either partial or radical nephrectomy, on the contralateral kidney within 12 months of the study, (3) are $<18$ years of age, (4) have a life expectancy $<3$ months as deemed by the treating physician, (5) are pregnant (confirmed by Beta-Human Chorionic Gonadotropin ( $\beta$ HCG test)) or (6) have known cold agglutinins.

\section{Study intervention}

The study intervention is renal hypothermia. For patients randomised to the hypothermia group, within $10 \mathrm{~s}$ of clamping the renal vessels, the kidney is encased with saline ice slush for a period of $10 \mathrm{~min}$ (the duration of slush encasement is based on available evidence and is in keeping with standard clinical practice ${ }^{23}{ }^{27}$ ). The slush is created from sterile saline using an operating room slush machine that is currently available at all participating trial locations. The slush is applied by the surgeon and lightly packed around the kidney to ensure complete coverage with at least a $2 \mathrm{~cm}$ layer. Slush removal is performed by the surgeon $10 \mathrm{~min}$ after the ice was applied. Enough slush is removed to uncover the tumour area. Surgical incision of the kidney begins as soon as the tumour area is uncovered. When reconstruction of the kidney is complete, the slush is completely removed from the surgical field and the clamps are removed from the renal vein and artery.

\section{Control intervention}

If the patient is randomised to no renal hypothermia, the tumour is removed immediately after all renal vessels are clamped. No hypothermia is the referent for comparison because it is considered the standard of care by many surgeons.

\section{Standard clinical care (applicable to both intervention and control)} Preoperative preparation consists of skin preparation and antibiotic prophylaxis, which are at the discretion of the operating surgeon. The type of anaesthetic agent is at the discretion of the anaesthesiologist. The incision (flank, subcostal or midline), dissection technique (intraperitoneal or extraperitoneal) and renal mobilisation are at the discretion of the operating surgeon. Five to $10 \mathrm{~min}$ prior to clamping the renal artery, $12.5 \mathrm{~g}$ of mannitol is administered intravenously. ${ }^{28}$ Renal vessels are occluded using vascular clamps of the surgeon's choice (vascular bulldog 


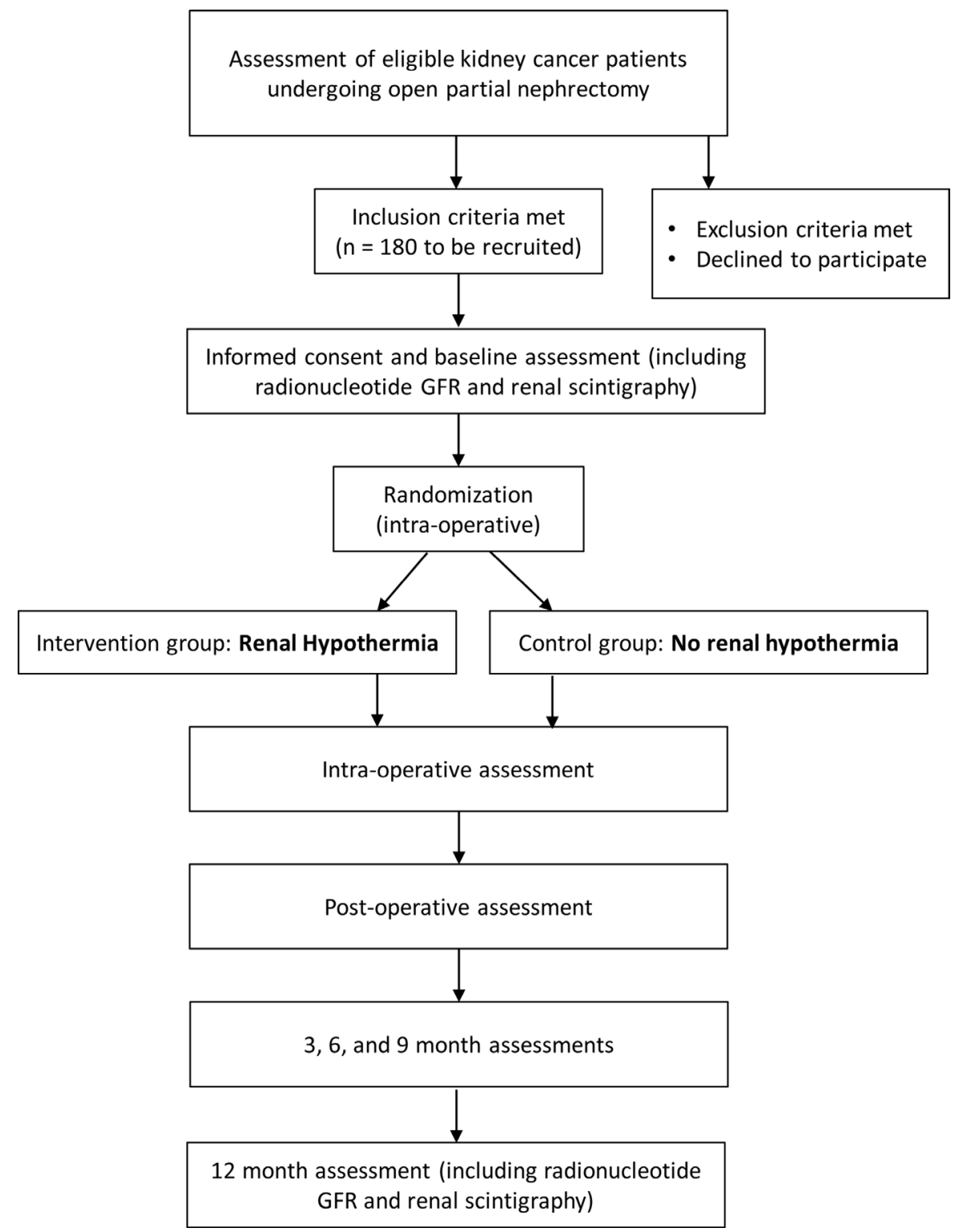

Figure 1 Study flow chart. GFR, glomerular filtration rate.

clamps, vascular Satinsky clamp, etc.). Occlusion of all renal arteries and veins must be achieved.

\section{Tumour resection and renal reconstruction}

The technique for tumour resection and renal reconstruction is at the discretion of the surgeon; however, the technique may be affected by type of ischaemia. In general, the renal capsule is scored with electrocautery delineating the parenchymal entry around the tumour. Using sharp dissection, the tumour is excised. A frozen section of the resection bed is not required. If the renal collecting system is entered, it is repaired using fine absorbable sutures. Grossly incised blood vessels are suture ligated and argon laser coagulation or electrocautery can be used to coagulate small vessels of the resection bed. Haemostatic agents may be applied, and the renal defect is repaired using oxidised cellulose bolsters and absorbable sutures.
Postrenal reconstruction

When the renal reconstruction is complete, vessel clamps are removed, and the kidney is inspected for haemostasis and reperfusion of the parenchyma. When the urologist is satisfied that adequate haemostasis is achieved, a drain is placed in the retroperitoneum adjacent to the kidney and the abdominal fascia and skin are reapproximated.

\section{Study outcomes}

Primary outcome

The primary outcome of the trial is overall GFR expressed in $\mathrm{mL} / \mathrm{min} / 1.73 \mathrm{~m}^{2}$. Overall renal function is measured via Technetium-99mTc-diethylenetriaminepentaacetic acid $\left({ }^{99 \mathrm{~m}} \mathrm{Tc}-\mathrm{DTPA}\right)$ plasma clearance 12 months postoperatively. 
Table 1 Timeline of study procedures and outcome assessments

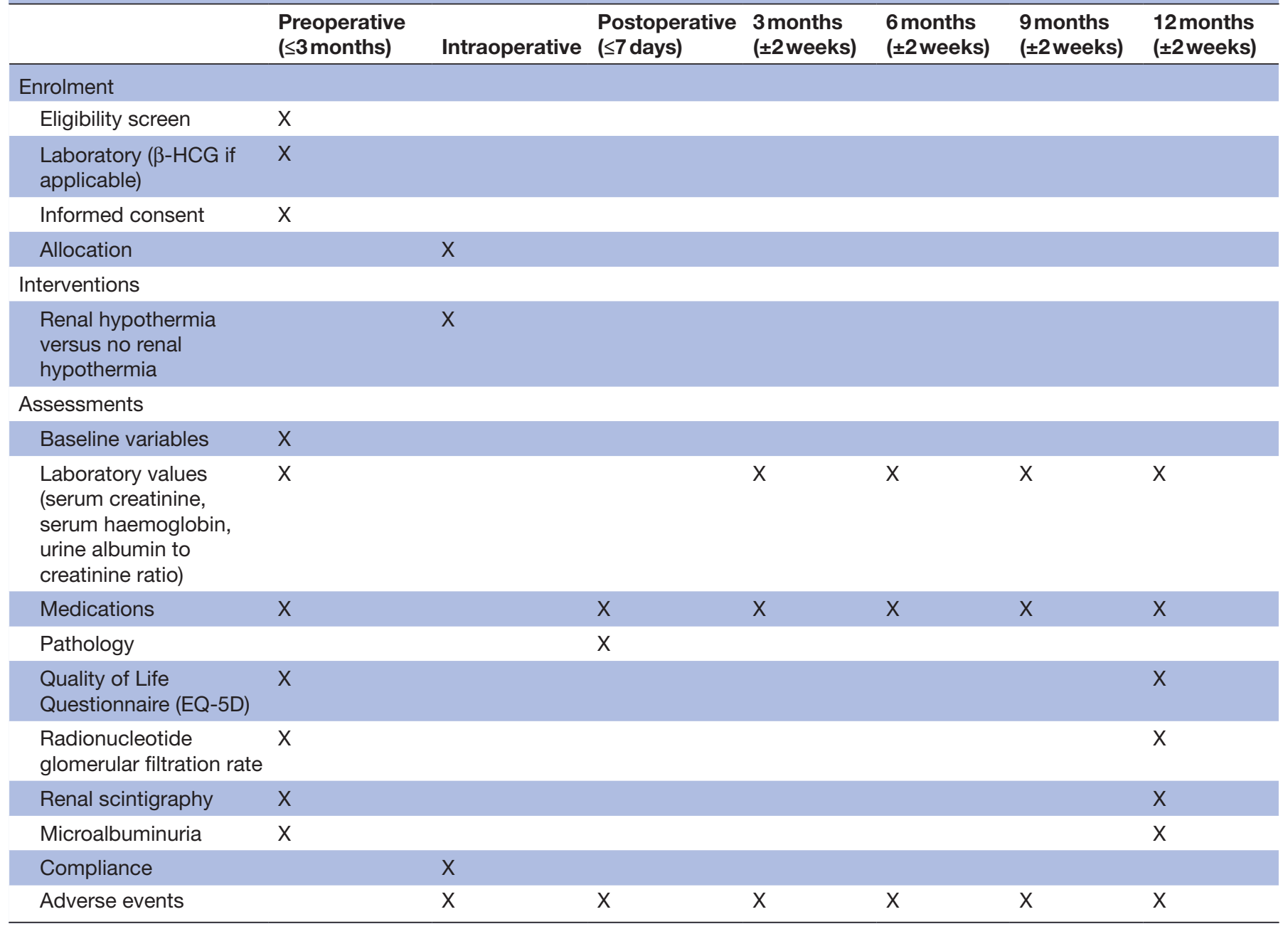

\section{Secondary outcomes}

A secondary outcome is affected GFR expressed in $\mathrm{mL} /$ $\min / 1.73 \mathrm{~m}^{2}$. Affected renal function is measured by the product of overall GFR (via ${ }^{99 \mathrm{~m}}$ Tc-DTPA plasma clearance) and relative contribution of the affected kidney (via renal scintigraphy) 12 months postoperatively.

In addition to the renal function assessment, we measure serum creatinine and spot urine albumin to creatinine ratios. We are also assessing the impact of intraoperative hypothermia on postoperative complications and exploring the associations between other variables such as patient and tumour characteristics on surgical outcomes. Data from the EuroQol Group Five-Dimensional (EQ-5D) Quality of Life questionnaire are collected to evaluate the quality of life of patients before and after partial nephrectomy.

Adverse events are collected each day while the patient is in hospital, on the first follow-up visit at 3 months and over the telephone at 6 and 9 months. Expected adverse events include: bowel ileus (abdominal distension $>4$ days with radiographic evidence associated with absent bowel sounds, nausea/vomiting and obstipation), wound infection (erythema and discharge at wound site with confirmed growth of infectious organisms on culture), urine leak (radiographic evidence of abdominal/pelvic fluid confirmed with fluid creatinine), retroperitoneal bleed (advanced trauma life support class II haemorrhage with radiographic evidence of blood in retroperitoneum), electrolyte abnormalities, dialysis, myocardial infarction (ECG changes, elevated troponins or imaging evidence of new loss of viable myocardium or new regional wall motion abnormality), deep vein thrombosis (confirmed by Doppler ultrasonography) and pulmonary embolus (confirmed by V/Q mismatch or pulmonary CT scan). All complications are graded according to the Clavien-Dindo surgical complication classification system.

\section{Frequency and duration of follow-up}

Data are collected preoperatively, during surgery, in the immediate postoperative period and at 3, 6, 9 and 12 months postoperative (table 1 ). At each data collection time point, the site research coordinator conducts a detailed review of the health record. In-person patient interviews are conducted at the preoperative, 3 and 12 months time points. The 6 and 9 months follow-ups are completed by telephone interview. Laboratory tests are drawn as per standard clinical care at each institution. Questionnaires are completed in person at baseline and 
12 months. If the patient reports any adverse events, the medical records are reviewed and abstracted. Case report forms (CRFs) are completed at each study time point and are supported fully by source documents (charts, reports, laboratory result sheets).

\section{Preoperative evaluation}

Demographic information includes age, gender, occupation, ethnicity and race. General quality of life is assessed using the EQ-5D health survey. A medication inventory is completed, and the patient's height and weight are measured. Blood, urine and radiographic test results are documented. The patient's overall renal function is assessed using ${ }^{99 \mathrm{~m}} \mathrm{Tc}-\mathrm{DTPA}$ plasma clearance and spot urine albumin to creatinine ratio. Side-specific (right and left) relative renal function is determined from renal scintigraphy. The preoperative evaluation including renal function assessment and laboratory evaluation are performed within 3 months of surgery.

\section{Perioperative and pathological data}

Perioperative information collected includes if bowel preparation was performed, antibiotic prophylaxis, venous thrombosis prophylaxis, diuretic administration, volume of intraoperative fluid replacement (crystalloid, colloid, blood products), duration of icing, arterial clamp time, blood pressure, presence of intraoperative hypotension (defined as a $20 \%$ relative decrease from preoperative systolic blood pressure), method of renal haemostasis and reconstruction. Pathology information includes tumour size, tumour weight, tumour histological subtype, tumour grade (for malignant tumours), tumour stage (for malignant tumours), nodal stage (for malignant tumours) and surgical margin status.

\section{2-month postoperative data (end of study)}

Quality of life, medications and laboratory information are documented at 12 months following surgery. A complication inventory is completed, and renal function is determined using the same tests as were performed preoperatively. The end of study evaluation occurs within fourteen days prior to or after 12 months from randomisation.

\section{Sample size}

Investigator consensus, expert consultation and a survey of urologists suggested that an absolute mean difference of $10.0 \mathrm{~mL} / \mathrm{min} / 1.73 \mathrm{~m}^{2}$ in GFR in favour of renal hypothermia is the minimal clinically important difference to change practice. In other words, urological surgeons would adopt or continue to perform renal hypothermia if a 10.0 unit or greater change in GFR was demonstrated. Likewise, they would likely discontinue the practice of renal hypothermia if less than a 10.0 unit change was demonstrated.

To detect a difference in overall renal function of $10.0 \mathrm{~mL} / \mathrm{min} / 1.73 \mathrm{~m}^{2}$ or greater between groups and a common $\mathrm{SD}$ of $20 \mathrm{~mL} / \mathrm{min} / 1.73 \mathrm{~m}^{2}$, we require 85 patients per arm for $90 \%$ power at $5 \%$ significance level.
To help guide our estimates, we relied on measures of renal function observed in patients from a prospective non-randomised study. ${ }^{12}$ We expect the overall average baseline GFR to be approximately $50-60 \mathrm{~mL} /$ $\min / 1.73 \mathrm{~m}^{2}$. To account for approximately $5 \%$ missing data, the total sample size required is estimated to be 180 patients (90 per arm).

\section{Recruitment}

We are implementing a number of initiatives to maintain recruitment, including regular tracking, monitoring and reporting of recruitment activity at each participating site, regular newsletters, progress reports and regularly scheduled investigator and research staff meetings. Participants have the right to withdraw from the study at any time, but are advised to discuss this decision with the site's qualified investigator or study coordinator before stopping the study. Information given to the research team before the participant withdraws consent may still be used in the analysis.

\section{Identification of patients}

Consecutive patients who choose partial nephrectomy and satisfy eligibility criteria are identified by the consultant urologist in the urological ambulatory care clinic. The urologist briefly describes the trial and determines if the patient is interested in participating. If the patient expresses interest, they are referred to the study coordinator who explains the protocol and potential risks and benefits in detail. If the patient chooses to enrol in the study, the study coordinator attains written informed consent and ensures all necessary preoperative information is documented.

\section{Patient and public involvement}

The renal hypothermia trial was developed in consultation with the Kidney Cancer Research Network of Canada (KCRNC; www.kcrnc.ca). The KCRNC is a collaborative of researchers, clinicians and patients with kidney cancer who are committed to facilitation and promotion of kidney cancer research in Canada. Since 2009, the KCRNC has hosted a research retreat that includes patients, researchers and clinicians. The KCRNC is actively involved in the development, implementation and promotion of this trial. The study design, intervention and outcomes were not thought to be a significant burden and were in line with patient preferences. When completed, the hypothermia trial results will be presented at the KCRNC meeting.

\section{Allocation of intervention \\ Randomisation}

Eligible and consenting patients are randomised using a central web-based randomisation system after the surgery has commenced and immediately prior to vascular clamping. Patients are allocated in a 1:1 ratio to one of the two study arms (renal hypothermia or no hypothermia) using permuted blocks of variable length (2 and $4)$, stratified by institution. Only the study statistician and 
a designate at the coordinating centre have access to the randomisation codes.

\section{Blinding}

To minimise sources of selection and ascertainment biases, patients, pathologists, radiologists, laboratory personnel and all members of the data safety and monitoring board (DSMB) are blinded to randomisation schemes and treatment administered. Since the interventions under investigation relate to the surgical conduct of partial nephrectomy, the surgeon and study coordinator conducting the intraoperative follow-up visit cannot be blinded. However, surgeons and coordinators are fully blinded prior to and during surgery until immediately before vascular clamping, thus reducing the risk of variable management between groups as a result of randomisation.

\section{Trial management}

The coordinating centre is located at The Ottawa Hospital and all data management is conducted by the Ottawa Methods Centre at the Ottawa Health Research Institute. Personnel at the coordinating centre include the study chair, research nurse coordinator, biostatistician and data analysts. The coordinating centre and the Ottawa Methods Centre work seamlessly to ensure proper data flow from screening to the end of study. The coordinating centre is responsible for the day-to-day management of the trial. Each site has a principal investigator and at least one research coordinator dedicated to this project. The site research coordinators have the responsibility to: obtain informed consent, attend the procedure and randomise patients via the web-based randomisation system, conduct all follow-up study visits and accurately fill out all data collection forms. The database is stored on the Ottawa Hospital Server under password protection and backed up daily. CRFs are stored in separate secure locations centrally and at each site. CRFs and the database are securely stored in separate locations for at least 15 years after trial completion. The primary investigators will have access to the data.

\section{Statistical methods}

All statistical comparisons will be performed with a two-sided 5\% significance level. Effect sizes will be presented with corresponding 95\% CIs. Analyses will be based on an intention-to-treat principle (ie, based on the participant's randomised study arm allocation irrespective of compliance with treatment allocation). As a complementary approach, we will also conduct a per protocol analysis for the renal function outcomes only.

\section{Analysis of primary outcome}

Absolute difference in overall GFR will be compared between groups using analysis of covariance (ANCOVA) and will be adjusted for treatment group and preoperative GFR. We will also analyse the change in overall GFR from baseline.
Analysis of secondary outcome

The analysis for difference in affected renal function will follow the same methods used for overall renal function.

Analysis of other outcomes

Mean differences in serum creatinine and spot albumin to creatinine ratio along with $95 \%$ CIs will be calculated. Complications and quality of life data (EQ-5D) will be analysed using $\chi^{2}$ tests and regression models adjusting for baseline characteristics that are statistically significantly different between groups. As an exploratory analysis, the impact of baseline GFR $(<45$ and $\geq 45 \mathrm{~mL} / \mathrm{min})$, level of proteinuria $(<50$ and $\geq 50 \mathrm{mg} / \mathrm{mmol})$, presence or absence of solitary kidney, patient age, clamp time, presence of intraoperative hypotension, tumour size and tumour location on renal function change will be assessed using multivariable linear regression adjusting for hypothermia. If necessary, data will be transformed to meet assumptions of common variance and approximate normality. Secondary analyses will also be conducted to investigate the influence of cointerventions, compliance and lost to follow-up on the robustness of the primary analysis.

\section{Frequency of analyses}

The main analysis will take place once follow-up is completed for all participants. No interim analysis will be performed. Adverse event data will be compiled by the study statistician and reviewed by a data safety monitoring committee when $50 \%$ of patients are accrued.

\section{Study monitoring}

The DSMB has full responsibility for the monitoring of response variables for adverse events throughout the trial. The committee functions independently from all other study committees and serves in an advisory role to the executive committee. The DSMB consists of three individuals, covering requisite expertise in clinical epidemiology, biostatistics, urological oncology and nephrology, and may consult external experts regarding specific outcome evaluations. Data are reviewed for safety every 6 months.

\section{Stopping rules}

No early stopping rules have been developed for this trial; early stopping criteria are at the discretion of the members of the independent DSMB who may make recommendations to terminate or continue the study. Early stopping of the study will only be based on appraisal of serious adverse events (SAEs) and is at the discretion of the clinical experts of the DSMB.

\section{Harms}

Adverse events are documented as part of the CRFs. Serious adverse events are submitted to the central coordinating centre using a study-specific reporting form. Adverse events are reported to local and central research ethics boards as per International Conference on Harmonisation/Good Clinical Practice (ICH-GCP) guidelines. 
Compliance with study allocation

In this study, compliance refers only to receiving the study intervention administered during the intraoperative period. We classify patients as being fully compliant if $10 \mathrm{~min}$ of renal hypothermia is administered, partially compliant if the patient is exposed to renal hypothermia for less than $10 \mathrm{~min}$ and non-compliant if the patient does not receive any planned renal hypothermia. Reasons for partial and non-compliance may stem from complications or death precluding the use of renal hypothermia. We expect partial/non-compliance to be less than $5 \%$ and therefore unlikely to influence the results. However, a secondary analysis will be performed to examine the influence of compliance on treatment effects through a per-protocol analysis. Any partial or non-compliance is captured in protocol deviation or protocol violation forms.

\section{Loss to follow-up}

We expect complete follow-up at 24 hours and hospital discharge given the complexity of care and necessary short-term follow-up. From past experience, we expect greater than $95 \%$ follow-up at 12 months. ${ }^{12}$

\section{Monitoring procedures}

An independent trained monitor performs all study monitoring at all participating sites. The monitor is trained on ICH-GCP, Tri-Council Policy Statement 2: Ethical Conduct for Research Involving Humans, study protocol, Ottawa Hospital Research Institute (OHRI) Standard Operating Procedures (SOPs) and study specific procedures.

\section{Site monitoring}

The trial data, compliance and adverse events are rigorously monitored using both remote and on-site methods of surveillance. This ensures that trial-related data are accurate, complete and verifiable from source documents and that participant rights and safety are protected. The monitor verifies compliance with the regulatory requirements, protocol, GCP, study-specific procedures and participant eligibility. In addition to evaluating the reported data for accuracy and completeness, the monitor identifies any trends in data that may be indicative of insufficient documentation or protocol deviations. Discrepancies noted in the data are recorded and the site is informed of all observations in the subsequent monitoring report.

The site monitor addresses deficiencies to the appropriate study team member in order to implement corrective actions or to recommend follow-up procedures. All observations noted during the monitoring visit appear in the monitoring report. The monitor assesses study files and documentation against ICH-GCP, regulatory requirements, protocol, OHRI SOPs and any study-specific SOPs.

\section{Interim monitoring visits}

To ensure patient safety and data integrity, regular remote monitoring visits are performed for each study site. If remote monitoring shows discrepancies in data or lack of compliance with regulations, or if requested by the DSMB or the site Investigator, on-site monitoring is performed. The study monitor assesses: CRF source data verification (SDV), patient eligibility and consent, study-specific SOPs, delegation logs, SAEs for recording and reporting completeness, regulatory documentation (for site and/or sponsor), training documents, protocol defined endpoints, essential document maintenance, deviation/violation recording and reporting, drug accountability, privacy considerations, and any protocol-specific procedures.

For remote monitoring, de-identified data from each site are sent to the monitor via secured courier. All data to be couriered are checked by the site coordinator prior to sending to ensure that patient privacy and confidentiality are maintained. No identifying materials are sent off-site. Documentation sent to the monitor for remote SDV includes: copies of signed and dated de-identified laboratory assessments, copies of signed and dated de-identified patient assessment forms (CRFs) with corresponding de-identified source documents, copies of de-identified source documentation that supports subjects eligibility to be enrolled into the study, copies of de-identified Investigator progress notes regarding patient-related decisions, signed and dated training logs as well as copies of materials used to train study staff (slide presentations, handouts).

\section{ETHICS AND DISSEMINATION}

Annual ethics renewal reports are submitted and reviewed by each study site. Once the trial is completed, results will be presented at an international research meeting and published in a peer-reviewed journal. Authorship eligibility will be in line with the International Committee of Medical Journal Editors' recommendations.

\section{DISCUSSION}

At the completion of the trial, we will better understand the benefits and risks of renal hypothermia during open partial nephrectomy. Specifically, we will know if renal hypothermia results in improved renal function at 12 months postoperative among patients undergoing open partial nephrectomy. Given that there are few exclusion criteria and multi-institutional involvement, we expect our findings to have broad generalisability. As this is the first randomised trial evaluating the use of renal hypothermia during open partial nephrectomy, the findings may have an immediate impact on patient care. If the study is negative, we can avoid the unnecessary use of renal hypothermia. If the study reveals a benefit of renal hypothermia, we will change our standard of practice regarding open partial nephrectomy procedures. A positive trial would also have a profound impact on laparoscopic partial nephrectomy as minimally invasive methods to achieve renal hypothermia have not been established. 


\section{TRIAL STATUS}

Since the trial opened in October 2012, 184 patients across 6 sites have been accrued and successfully randomised. We expect data collection to be complete by November 2018. To date, no SAEs have been reported that were considered related to the study intervention.

\section{Author affiliations}

${ }^{1}$ Division of Urology, Department of Surgery, The Ottawa Hospital, Ottawa, Ontario, Canada

${ }^{2}$ Clinical Epidemiology Program, Ottawa Hospital Research Institute, Ottawa, Ontario, Canada

${ }^{3}$ Division of Urology, Departments of Surgery and Surgical Oncology, Princess Margaret Cancer Centre, University Health Network and the University of Toronto, Toronto, Ontario, Canada

${ }^{4}$ Department of Urology, Mayo Clinic, Rochester, Minnesota, USA

${ }^{5}$ Oxford Clinical Trial Research Unit, University of Oxford, Oxford, UK

${ }^{6}$ Division of Urology, McMaster University, Hamilton, Ontario, Canada

${ }^{7}$ Division of Urology, Université Laval, Quebec City, Quebec, Canada

${ }^{8}$ Division of Urology, Department of Surgery, Western University, London, Ontario,

Canada

${ }^{9}$ Department of Urology, Dalhousie University, Halifax, Nova Scotia, Canada

Acknowledgements Many thanks to the clinicians, researchers and patients of the Kidney Cancer Research Network of Canada for their contributions to the development of this trial.

Contributors RHB, IC, GK, CM, RM, AF, MJ, BCL, JC, AK, FP, JI, RR and DAF contributed to the conception and design of the study. RHB, IC, GK, AF, MJ, BCL, JC, $\mathrm{LL}, \mathrm{AK}, \mathrm{FP}, \mathrm{Jl}, \mathrm{RR}$ and DAF were involved in obtaining funding. RHB, IC, CM, SC, LTL, RM and LL contributed to data collection and/or analysis. All authors were involved in drafting the manuscript. All authors read and approved the final manuscript.

Funding This work was supported by the Canadian Institutes of Health Research (CIHR) grant number MOP-110993.

Competing interests $\mathrm{CM}$ has been an adviser for Abbvie, Astellas, Ferring, Janssen and Sanofi; and has participated in clinical trials supported by Abbvie. LTL has been an adviser for Ferring and Sanofi; and has received a grant from Sanofi. IC has been an adviser for Abbvie and Ferring; and has received speaker honoraria from Abbvie, Acerus and Ferring. The remaining authors report no competing personal or financial interests.

Patient consent Not required.

Ethics approval Ethics approval was obtained for all participating study sites: Ottawa Health Science Network Research Ethics Board (The Ottawa Hospital, Ottawa; Protocol ID 2010767-01H); Ontario Cancer Research Ethics Board (University Health Network, Toronto; Protocol ID 12-011); Hamilton Integrated Research Ethics Board (St. Joseph's Hospital, Hamilton; Protocol ID 12-3779); Comité d'éthique et de la recherche du CHU de Québec-Université Laval (Centre Hospitalier Universitaire (CHU) de Québec, Quebec City; Protocol ID 2012-1040); Western University Health Science Research Ethics Board (London Health Sciences Centre, London; Protocol ID 103164) and Nova Scotia Health Authority Research Ethics Board (Capital District Health Authority, Halifax; Protocol ID 1015028).

Provenance and peer review Not commissioned; externally peer reviewed.

Open access This is an open access article distributed in accordance with the Creative Commons Attribution Non Commercial (CC BY-NC 4.0) license, which permits others to distribute, remix, adapt, build upon this work non-commercially, and license their derivative works on different terms, provided the original work is properly cited, appropriate credit is given, any changes made indicated, and the use is non-commercial. See: http://creativecommons.org/licenses/by-nc/4.0/.

\section{REFERENCES}

1. Society AC. Cancer statistics centre. 2017. https://cancerstatistics center.cancer.org/?_ga=2.143854581.1765154383.15127614061176818792.1512576926\#!/data-analysis/IncidenceTrend (cited 8 Jan 2018).
2. Frank I, Blute ML, Cheville JC, et al. Solid renal tumors: an analysis of pathological features related to tumor size. J Urol 2003;170:2217-20.

3. Society AC. Cancer statistics center. 2017. https://cancerstatistics center.cancer.org/? ga =2.143854581.1765154383.15127614061176818792.1512576926\#!/ (cited 4 Dec 2017).

4. Annual Meeting of the Society of Urologic Oncology. In: Cooperberg M, Kane C, Mallin K, Carroll P, et al. eds. National trends in treatment of stage I renal cell carcinoma. Bethesda, Maryland: Annual Meeting of the Society of Urologic Oncology, 2008.

5. Herr HW. Partial nephrectomy for unilateral renal carcinoma and a normal contralateral kidney: 10-year followup. J Urol 1999;161:33-5.

6. Lau WK, Blute ML, Weaver AL, et al. Matched comparison of radical nephrectomy vs nephron-sparing surgery in patients with unilateral renal cell carcinoma and a normal contralateral kidney. Mayo Clin Proc 2000;75:1236-42.

7. McKiernan J, Simmons R, Katz J, et al. Natural history of chronic renal insufficiency after partial and radical nephrectomy. Urology 2002;59:816-20.

8. Lee CT, Katz J, Shi W, et al. Surgical management of renal tumors 4 $\mathrm{cm}$. or less in a contemporary cohort. J Urol 2000;163:730-6.

9. Dash A, Vickers AJ, Schachter LR, et al. Comparison of outcomes in elective partial vs radical nephrectomy for clear cell renal cell carcinoma of 4-7 cm. BJU Int 2006;97:939-45.

10. Huang WC, Levey AS, Serio AM, et al. Chronic kidney disease after nephrectomy in patients with renal cortical tumours: a retrospective cohort study. Lancet Oncol 2006;7:735-40.

11. Leibovich BC, Blute M, Cheville JC, et al. Nephron sparing surgery for appropriately selected renal cell carcinoma between 4 and $7 \mathrm{~cm}$ results in outcome similar to radical nephrectomy. J Urol 2004;171:1066-70.

12. Clark AT, Breau RH, Morash $\mathrm{C}$, et al. Preservation of renal function following partial or radical nephrectomy using 24-hour creatinine clearance. Eur Urol 2008;54:143-52.

13. Thompson RH, Boorjian SA, Lohse CM, et al. Radical nephrectomy for pT1a renal masses may be associated with decreased overall survival compared with partial nephrectomy. J Urol 2008;179:468-73.

14. Go AS, Chertow GM, Fan D, et al. Chronic kidney disease and the risks of death, cardiovascular events, and hospitalization. $N$ Engl $J$ Med 2004;351:1296-305.

15. Lavallée LT, Tanguay S, Jewett MA, et al. Surgical management of stage T1 renal tumours at Canadian academic centres. Can Urol Assoc J 2015;9:99.

16. Jewett MA, Rendon R, Lacombe $L$, et al. Canadian guidelines for the management of small renal masses (SRM). Can Urol Assoc $J$ 2015;9:160.

17. Campbell S, Uzzo RG, Allaf ME, et al. Renal Mass and localized renal cancer: AUA guideline. J Urol 2017;198:520-9.

18. Ljungberg $\mathrm{B}$, Bensalah $\mathrm{K}$, Canfield $\mathrm{S}$, et al. EAU guidelines on renal cell carcinoma: 2014 update. Eur Urol 2015;67:913-24.

19. Gill IS, Kavoussi LR, Lane BR, et al. Comparison of 1,800 laparoscopic and open partial nephrectomies for single renal tumors. $J$ Urol 2007:178:41-6.

20. Thompson RH, Frank I, Lohse CM, et al. The impact of ischemia time during open nephron sparing surgery on solitary kidneys: a multiinstitutional study. J Urol 2007;177:471-6.

21. Lane BR, Babineau DC, Poggio ED, et al. Factors predicting renal functional outcome after partial nephrectomy. $J$ Urol 2008;180:2363-9.

22. Bogardus GM, Schlosser RJ. The influence of temperature upon ischemic renal damage. Surgery 1956;39:970-4.

23. Ward JP. Determination of the Optimum temperature for regional renal hypothermia during temporary renal ischaemia. $\mathrm{Br} J$ Urol 1975;47:17-24

24. Novick AC. Renal hypothermia: in vivo and ex vivo. Urol Clin North Am 1983;10:637-44.

25. Rimmer L, Fok M, Bashir M. The history of deep hypothermic circulatory arrest in thoracic aortic surgery. Aorta 2014;2:129-34.

26. Li LR, You C, Chaudhary B. Intraoperative mild hypothermia for postoperative neurological deficits in people with intracranial aneurysm. Cochrane Database Syst Rev 2016;3:CD008445-CD.

27. Uzzo RG, Novick AC. Nephron sparing surgery for renal tumors: indications, techniques and outcomes. J Urol 2001;166:6-18.

28. Aydin G, Okiye SE, Zincke H. A comparative study of several agents alone and combined in protection of the rodent kidney from warm ischaemia: methylprednisolone, propranolol, furosemide, mannitol, and adenosine triphosphate-magnesium chloride. Urol Res 1983;11:105-9. 